\section{Immunohistochemical and ultrastructural visualization of different routes of oocyte elimination in adult rats}

\author{
M.L. Escobar Sánchez, O.M. Echeverría \\ Martínez, G.H. Vázquez-Nin \\ Departamento de Biología Celular, \\ Facultad de Ciencias, Universidad \\ Nacional Autónoma de México (UNAM), \\ Coyoacán, México
}

\section{Abstract}

Cell death is a process for maintaining homeostasis in tissues and organs. In the ovary, apoptotic cell death has been implicated in follicular atresia; in the elimination of the follicles that are not ovulated during adult life. Recent studies indicate that apoptosis and autophagy are two programmed processes of cell death. Apoptosis is performed by proteases called caspases and leads to such morphological traits as DNA fragmentation. Autophagy, in turn, is characterized by the exacerbated formation of autophagosomes; a process in which the amount of the LC3 and Lamp 1 proteins increases. In this study, oocytes from all stages of the estrous cycle of Wistar rats were analyzed. The apoptosis process was identified by immunodetecting active Caspase- 3 and locating DNA fragmentation using the TUNEL technique. Autophagy was evaluated through immunodetection of the LC3 and Lamp 1 proteins, and by ultrastructural localization of autophagic vesicle formation. All techniques were conducted using the same oocytes. Results show that all phases of the estrous cycle contain dying oocytes that test positive simultaneously for apoptosis and autophagy markers. The highest level of apoptosis was found during estrus; while the proestrous stage had the highest level of autophagy. The diestrous and metestrous phases were characterized by a high frequency of the presence of markers of apoptosis and autophagy in the same oocyte. Our results demonstrate that during oocyte elimination in adult rats the proteins involved in both processes, apoptosis and autophagy, are present in the same cell at the same time.

\section{Introduction}

Female mammals are born with a defined set of oocytes located in the functional structure of the ovary: that is follicles. The oocytes that are selected for release constitute less than $1 \%$ of the total; the remainder is removed via the process of follicular atresia,,${ }^{1,2}$ which is an important, selective degenerative process that takes place in the gonads of mammals both before and after birth. ${ }^{3}$

Follicular development is a dynamic process that involves the growth and development of follicles in different phases: primordial, primary, secondary (or pre-antral), and antral follicles. In adult organisms, follicular development and granulosa cell differentiation are regulated by several autocrine or paracrine factors, such as steroids, gonadal peptides and growth factors. During the early stages of follicular development, the paracrine factors act as a mitogen, whereas in later stages they enhance granulosa cell differentiation and luteinization in a coordinated manner with gonadotropin-cAMP-generated signals. ${ }^{4}$

Follicular atresia occurs following different patterns depending on the exact follicular phase: in its early stages (primordial, primary, small pre-antral), atresia is initiated by oocyte apoptosis that causes the death of granulosa cells. ${ }^{3}$ However, in late pre-antral, antral and preovulatory follicles, atresia is triggered by granulosa cell death. ${ }^{5}$ Follicular atresia is characterized by increased apoptotic cell death. The morphological and biochemical characteristics of apoptosis include nuclear and cytoplasmic condensation and the break-up of membrane-bound fragments called apoptotic bodies. ${ }^{6}$ Biochemically, cleavage of DNA, caspase-mediated proteolysis of specific intracellular proteins, and the flipping of phosphatidylserine from the inner to the outer leaflet of the plasma membrane, are the hallmarks of apoptosis. The caspases include both initiator (-8 and -9$)$ and effector types $(-3,-6$, and -7 ) that, when activated, cleave the enzymes and proteins that are essential for cell metabolism. ${ }^{7}$ Recent reports indicate that in addition to apoptosis, autophagy also participates as a programmed cell death process. Autophagic cell death is characterized by the presence of double, or multiple, membrane cytoplasmic vesicles that engulf bulk cytoplasm and/or cytoplasmic organelles, such as mitochondria and the endoplasmic reticulum. Autophagy begins by enclosing cytoplasmic constituents in a membrane sac, resulting in the formation of double membrane structures called autophagosomes. ${ }^{8}$ The autophagic vesicles and their contents are then destroyed by the lysosomal hydrolases in the eukaryotic cells.

The molecular mechanisms governing the performance and regulation of the autophagic program involve several autophagy-specific genes (Atg), elements that are highly conserved among species as distinct as humans and yeast. ${ }^{9}$ The microtubule-associated protein
Correspondence: Dr. Gerardo Hebert VázquezNin, Universidad Nacional Autónoma de México, Ciudad Universitaria, Delegación Coyoacán C.P. 04510, México.

Tel. +521.55 .56224881 - Fax: +521.55 .56224828 .

E-mail: vazqueznin@ciencias.unam.mx

Key words: atresia, apoptosis, autophagy, oocyte, estrous cycle.

Contributions: all authors contributed equally to the work.

Acknowledgments: the authors would like to thank Ernestina Ubaldo Pérez for technical assistance. They also thank Paul C. Kersey Johnson for reviewing the English word usage and grammar.

This work was supported by PAPITT IN212912-2.

Received for publication: 20 December 2011. Accepted for publication: 8 February 2012.

This work is licensed under a Creative Commons Attribution NonCommercial 3.0 License (CC BYNC 3.0).

(C) Copyright et al., 2012

Licensee PAGEPress, Italy

European Journal of Histochemistry 2012; 56:e17 doi:10.4081/ejh.2012.e17

light chain 3 (LC3) is the mammalian homologue of yeast $\operatorname{Atg}^{8,10}$ and is involved in autophagic vesicle formation. Lysosomes are acidic vesicles that participate in the breakdown of diverse macromolecules and are also implicated in the catabolism of cytoplasmic components through autophagy. ${ }^{11}$ The most abundant proteins found in lysosomal membranes are the lysosome-associated membrane proteins 1 and 2 (Lamp 1 and Lamp 2), which together constitute almost $50 \%$ of all lysosomal membrane proteins. Under optimal conditions, cells ensure the turnover of old and damaged organelles by maintaining a low basal level of autophagic flow. However, when autophagic activity increases over the basal level the process of cellular death begins.

The expression of several apoptotic proteins has been identified in the ovary; for instance, the increased expression of the death factor Bax, a Bcl-2 family member, in granulosa cells has been correlated with apoptosis in the rat.12 In mice oocytes and granulosa cells, caspase-9 is required for apoptosis, ${ }^{13}$ while caspase 2 is necessary for the apoptosis induced by anticancer drugs and during development. ${ }^{14}$ Protease caspase- 3 has been identified in oocytes, ${ }^{15}$ and it has been suggested that several caspases are activated in oocytes prior to cellular budding and fragmentation. ${ }^{16}$ Also, apoptosis has long been considered the principal mechanism for oocyte depletion in both normal and pathological conditions. As men- 
tioned above, follicular atresia has been attributed to apoptotic cell death; however, recent reports have demonstrated that autophagy is also an important process during oocyte death in prepubertal mammals. Moreover, cellular elimination in these animals involves both types of cell death. ${ }^{17-19}$ The aim of the present study is to determinate whether the proteins involved in apoptotic cell death and autophagic cell death are present in the adult oocytes, and to evaluate the ultrastructural characteristics of the oocytes during the follicular atresia. Lamp-1, LC3, active-caspase-3 proteins, and TUNEL were identified in oocytes using immunohistochemical techniques. The ultrastructural features were evaluated by means of electron microscopy. We found that the proteins involved in autophagic and apoptotic cell death are present in the same cell at the same time throughout the estrous cycle.

\section{Materials and Methods}

\section{Experimental animals}

A total of 60 adult Wistar rats were used in this study in three different assays. They were kept under a $12 \mathrm{~h}$ light-darkness cycle with water and food ad libitum. Five regular estrous cycles were controlled by means of vaginal smears before sacrifice. All animals were handled in accordance with the NIH Guide for the Care and Use of Laboratory Animals.

\section{Ovary tissue processing for optical microscopy}

Ovaries were fixed in $2 \%$ paraformaldehyde in phosphate-buffered saline (PBS) at $\mathrm{pH} 7.2$ for $2 \mathrm{~h}$ at room temperature, embedded in paraffin, and serially sectioned $(5 \mu \mathrm{m})$. The sections from each ovary were aligned on glass microscope slides covered with poli-l-lysine (Sigma, Toluca, Mexico), and observed under a Nikon Eclipse E600 Microscope. 96 serial sections of adult rat ovaries of each phase of the estrous cycle (diestrous, metestrous, proestrous, and estrus) were studied. Images were recorded with a Nikon Digital Camera DXM1200F and processed using the Image Processing and Analysis Java (ImageJ) program. ${ }^{20}$

\section{Ovary tissue processing for elec- tron microscopy}

Ovaries were fixed in $2.5 \%$ glutaraldehyde$4 \%$ formaldehyde in phosphate-buffered saline (PBS) at $\mathrm{pH} 7.2$ for $2 \mathrm{~h}$. After rinsing in the same buffer, tissues were post-fixed in $1 \%$ osmium tetroxide $\left(\mathrm{OsO}_{4}\right)$ in a $\mathrm{PBS}$ buffer at $\mathrm{pH}$ 7.2 , for $1 \mathrm{~h}$. Tissues were then dehydrated using a graded series of ethanol and embedded in Epon (Embed-812, Electron Microscopy Science, Hatfield, PA, USA). Semi-thin sections were stained with toluidine blue. Selected areas were thin-sectioned, and grids were counterstained with uranyl acetate and lead citrate. Sections were examined under a JEOL 1010 electron microscope operated at 100 KV. Digital images were taken with a Hamamatsu camera.

\section{TUNEL procedure}

Evaluation of DNA fragmentation was performed with an Apoptag Red In Situ Apoptosis Detection Kit (Millipore Corp., Billerica, MA, USA). Sections were treated with proteinase $K$ for $15 \mathrm{~min}$ at room temperature, and then
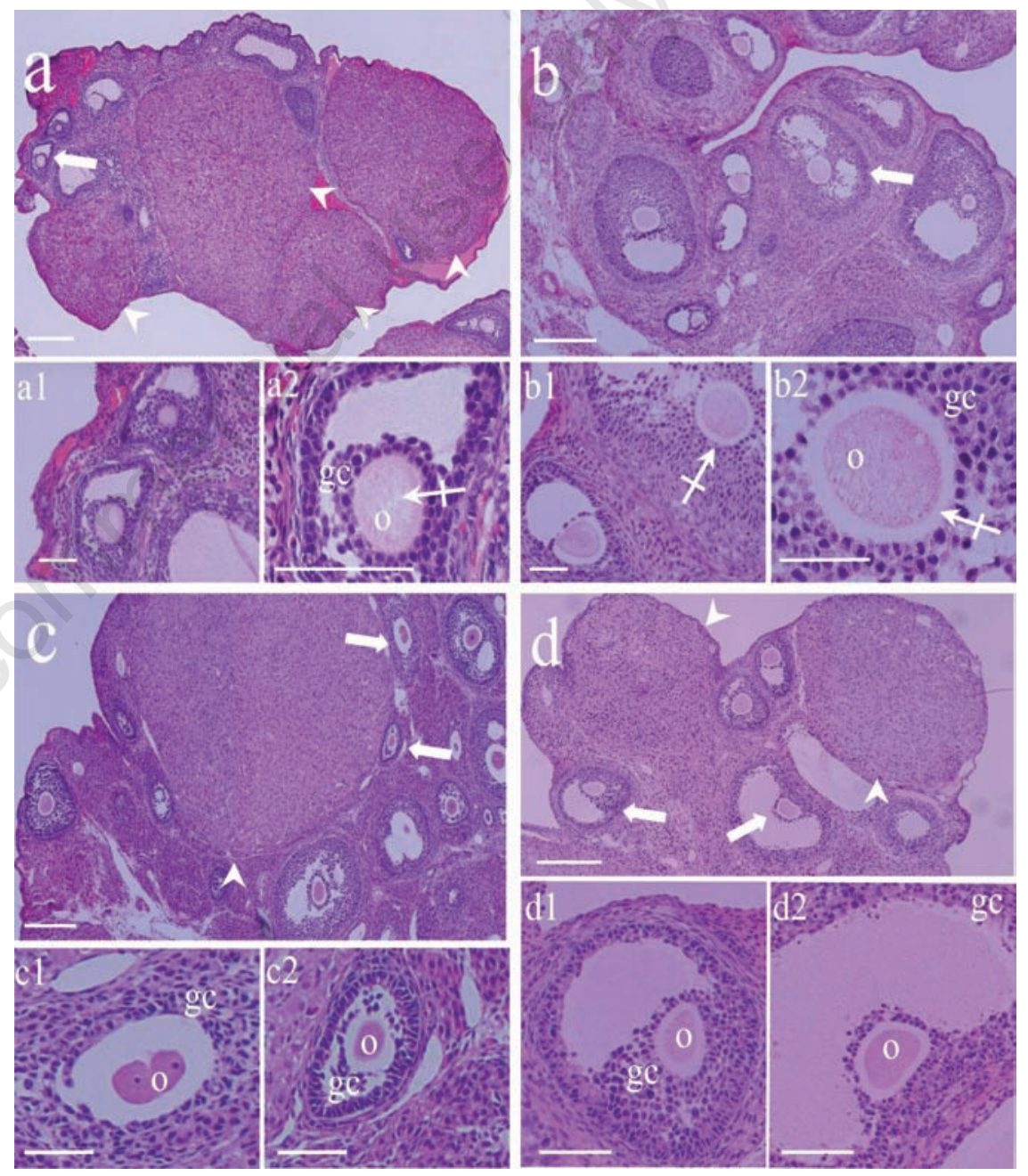

Figure 1. Morphological characteristics of rat ovaries: a) metestrous; b) diestrous; c) proestrous; and, d) estrus. The ovaries presented different morphological patterns; in the ovaries in metestrous the corpora lutea was more evident than in the other phases of the cycle (arrowheads), Atretic follicles with different degrees of degeneration were observed (arrows) in all phases of the cycle. Under high magnifications (a1, a2, b1, b2, c1, c2, d1, and $\mathrm{d} 2$ ), all follicles are in different stages of the process of atresia and show altered oocytes with irregular shapes and cytoplasmic regions with clear vesicles (crossed arrows). The oocyte in $\mathrm{cl}$ is segmented in two nucleated pseudo-blastomers with large nucleoli. $o$, oocyte; $g c$, granulosa cells. Scale bars: a, b, c, and d, 200 microns; all others $50 \mu \mathrm{m}$. 
sue sections in citrate-buffer $0.1 \mathrm{M}, \mathrm{pH} 6$ (BioGenex, Fremont, CA, USA) in a Panasonic oven for $3 \mathrm{~min}$ at $1300 \mathrm{~W}$, and $6 \mathrm{~min}$ at $780 \mathrm{~W}$. After cooling, tissue sections were washed in phosphate-buffered saline (PBS) and then incubated with primary antibody anti-active caspase-3 (1:200 in PBS dilution), anti-Lamp1 (1:100 in PBS dilution), or anti-LC3 (1:200 in PBS dilution) for $18 \mathrm{~h}$ at $4^{\circ} \mathrm{C}$. The negative controls were carried out omitting the primary antibodies. After washing, slides were incubated with an anti-rabbit immunoglobulin coupled to Alexa fluor 488 (Invitrogen) for $1 \mathrm{~h}$ in darkness at room temperature. After that, the preparations were washed and counterstained with DAPI (Sigma) to evaluate DNA distribution. Slides were covered with mounting media for fluorescence microscopy (Vectashield Mounting Medium).

\section{Western blot analysis}

In order to evaluate the specificity of the antibodies Western blotting analyses were carried out. Adult rat ovaries were sectioned and placed during $15 \mathrm{~min}$ in lysis buffer $(50 \mathrm{mM}$ Tris-Cl, pH 7.5; $150 \mathrm{mM} \mathrm{NaCl}, 0.1 \%$ SDS, $1 \mathrm{mM}$ PMSF, $0.5 \%$ sodium deoxycholate, and $1 \%$ Nonidet P-40) supplemented with protease inhibitor cocktail complete (Roche, Mannheim, Germany). The total proteins were measured by Bradford Assay; $50 \mu \mathrm{g}$ total proteins were loaded on a $12 \%$ SDS-PAGE gel. Proteins were transferred to polyvinylidene fluoride (PVDF) membranes, which were incubated $1 \mathrm{~h}$ at room temperature in blocking buffer. Then the membranes were incubated with anti-active caspase 3, anti-Lamp-1, and anti-LC3 antibodies (Sigma, Abcam and Affinity Bioreagents, respectively) in a dilution 1:500. The proteins were successively tagged by incubation with peroxidase-conjugated secondary antibody (Jackson, Newmarket, UK) 1:10,000 in blocking buffer during $1 \mathrm{~h}$ at room temperature. Using Horse Radish Peroxidase (HRP) as substrate (Immobilon Western, Millipore $\mathrm{Co}$, USA), the specific labeling was detected by chemiluminesce. The film Hyperfilm Amersham Biosciences was expossed to the membranes in order to detect the chemiluminesce.

\section{Statistical analysis}

Fluorescent immunodetections of Lamp1 and LC3 proteins were carried out using the ImageJ program. The fuorescent signal incorporated in the cytoplasm of the cells was evaluated by the pixel intensity in 650 images obtained from 60 rats. The background of the slide was evaluated with Image J program tools. There was no discrimination between follicular phases: i.e., all oocytes were considered in this evaluation. They were divided into two groups according to the type of follicle: atretic or healthy. A Student t-test was used to assess the statistical significance of the differences $(\mathrm{P}<0.05)$.

\section{Results}

\section{Morphological analysis}

Sections from the ovaries of rats during the metestrous, diestrous, proestrous, and estrus phases were analyzed to evaluate the morphological characteristics of their follicles. Follicle types in the process of atresia were observed in each ovary. The atretic follicles were classified according to the following morphological characteristics: altered shape of the follicle, granulosa cells detached from the oocyte, apoptotic signs in the granulosa cells, altered shape of the oocyte, and altered cytoplasmic organelles in the oocyte.

Follicles from all four phases of the cycle showed atretic traits, including an altered oocyte. The oocytes showed noticeably clear cytoplasmic vesicles and signs of degradation (Figure $1 \mathrm{a}, \mathrm{b}$; high magnification). The cytoplasm of some oocytes is segmented, and some segments have fragments of highly compacted nuclei. The junctions between granulosa cells become scarce or were eliminated (Figure 1 c,d).

\section{Evaluation of autophagy and apop- tosis}

The apoptotic process was studied using the TUNEL assay to detect DNA fragmentation, and active caspase-3 immunodetection. The autophagic process was identified by quantitative immunolabeling of LC3 and Lamp1, and measuring the fluorescence intensity. The levels of labeling in the cytoplasmic space of Lamp1 and LC3 were significantly different between the cells that were positive and negative, respectively, for the autophagic cell death markers (Figure 2).

To discriminate between the basal autophagic process and autophagic cell death, fluorescence intensity was measured. We analyzed the fluoresce intensity of altered oocytes morphologically considered as autophagic and non-autophagic. A marked increased in the expression of Lamp1 and LC3 was found in the oocytes dying by a predominantly autophagic process in contrast to those which are undergoing a non-autophagic death process. Statistical significance was determinate by means of Student t-test. The fluorescence intensity of the altered oocytes is significantly higher than that of the unaltered ones $(\mathrm{P}<0.05$; Figure $3 \mathrm{a}, \mathrm{b})$. The oocytes in the four phases of the estrous cycle analyzed showed different labeling patterns, including cells positive for the autophagic markers, Lampl and LC3 (Figure 4), and others positive for the apoptotic markers, active caspase- 3 and TUNEL (Figure 5). However, a significant number of oocytes were positive for the markers of autophagy and apoptosis; i.e., Lamp1 LC3, active caspase- 3 , and the TUNEL technique (Figure 6). Indeed, over $40 \%$ of cells exhibited simultaneous markers of apoptotic and autophagic cell death in all phases of the estrous cycle (Figure 7). Signs of both apoptosis and autophagy were present at the same time in the same cells in all phases of the estrous cycle. The highest frequency of this labeling pattern was found in the diestrous and

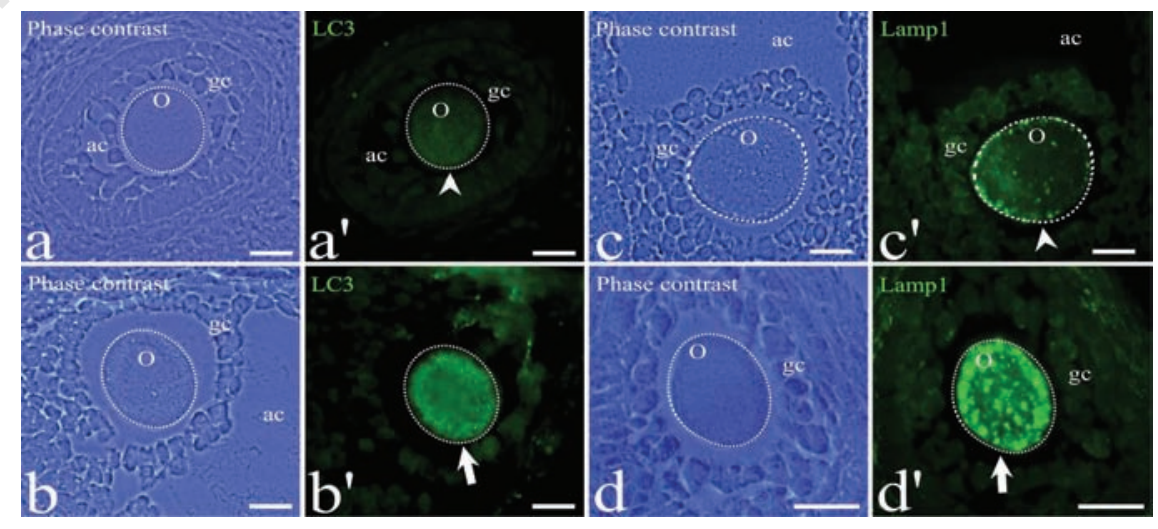

Figure 2. Immunodetection of the pro-autophagic proteins LC3 and Lamp1 in atretic oocytes in early antral (a), antral follicles (b and c), and secondary (d). The phase contrast shows the general morphology of the follicles. The green fluoresce indicate the both proteins Lamp1 and LC3 immunodetection. Some granulosa cells (gc) are positive for Lamp1 and LC3. The chromatin of the cells is labeled in blue by DAPI staining method. The dotted circles indicate the approximate shape of the oocyte. Arrowheads point to the oocytes with low labeling intensity; arrows indicate highly labeled oocytes in the process of cell death. $o$, oocyte; $g c$, granulosa cells; $a c$, antral cavity. Scale bars 20: $\mu \mathrm{m}$. 
metestrous stages, while the lowest was found during estrus.

To evaluate the specificity of the three antibodies used, active caspase-3, Lamp1, and LC3, Western Blot analyses of the total proteins of the adult rat ovary were performed. The results indicate that the antibodies recognize the corresponding proteins. The anti-active caspase- 3 recognizes $\mathrm{a} \approx 20 \mathrm{kDa}$ protein corresponding to the p20 subunit of the active caspase- 3 . The anti-LC3 recognizes the two isoforms of this protein: LC3-I (18kDa protein) and LC3-II (16 $\mathrm{kDa}$ protein). The anti-Lampl recognizes a $\approx 120 \mathrm{kDa}$ protein (Figure $8 \mathrm{a}$ ). The negative controls of the antibodies used in the present study were performed by omitting the primary antibody (Figure 8b). The phase contrast images show altered oocytes in follicles in different phase of development; the absence of mark is evident in the images of the corresponding field (Figure 8b).

\section{Ultrastructural features}

Electron microscopic analysis showed differences between normal and altered oocytes. Normal oocytes maintained a close relation with the somatic cells, their cytoplasm was homogeneous, contained small quantities of autophagic vesicles, and the chromatin was sparsely distributed in the nucleoplasm. In the altered oocytes, however, the relation with the granulosa cells was progressively lost and the chromatin began to condense. In all follicular phases some of the oocytes in the process of autophagic cell death showed abundant autophagic vesicles (Figure 9). The autophagic cell death could be observed in all stages of development of the follicles until selectable ones. One of the characteristics observed in the oocytes dying in different phases of the estrous cycle was cellular segmentation, which is a feature typical of apoptosis (Figure 10a). It is interesting to note that some of the cellular segments had diverse cytoplasmic content that include the nucleus or fragments of nucleus that often contained a highly-compacted nucleolus. Another ultrastructural characteristic of the oocytes during the process of autophagic cell death was the presence of a high number of vacuoles with, or without organelles (Figure 10b). An interesting ultrastructural characteristic of the autophagic process in adult oocytes was the presence of marked cellular membrane reorganization. The cytoplasm has numerous dispersed autophagic vesicles that are easily observable under high magnification (Figure 11 a,b,c). Many of these autophagic vesicles contain cytoplasmic structures in different phases of degradation, including some membrane debris. It is important to note that these characteristics were present in oocytes obtained from rats in the four phases of the estrous cycle (Figure 12).

\section{Discussion}

Follicular atresia is an important event, whose principal functions consist in maintaining homeostasis in the ovary and contributing to the ovulation of functional, healthy oocytes. Adult rats ovulate every four days, thus allow-

a

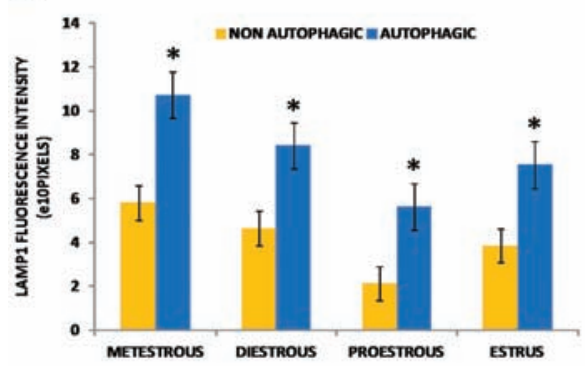

b

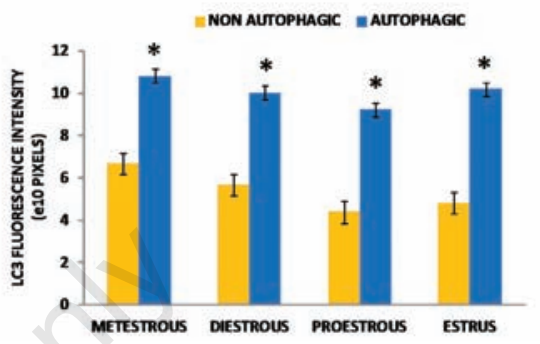

Figure 3. Quantification of the fluorescence intensity of Lamp1 (a) and LC3 (b). There is a significant difference in the intensity of the immunodetection of these two proteins between autophagic and non-autophagic cells. Error bars represent standard error and the asterisk indicate the significant differences obtained by a Student $t$-test $(P<0.05)$.
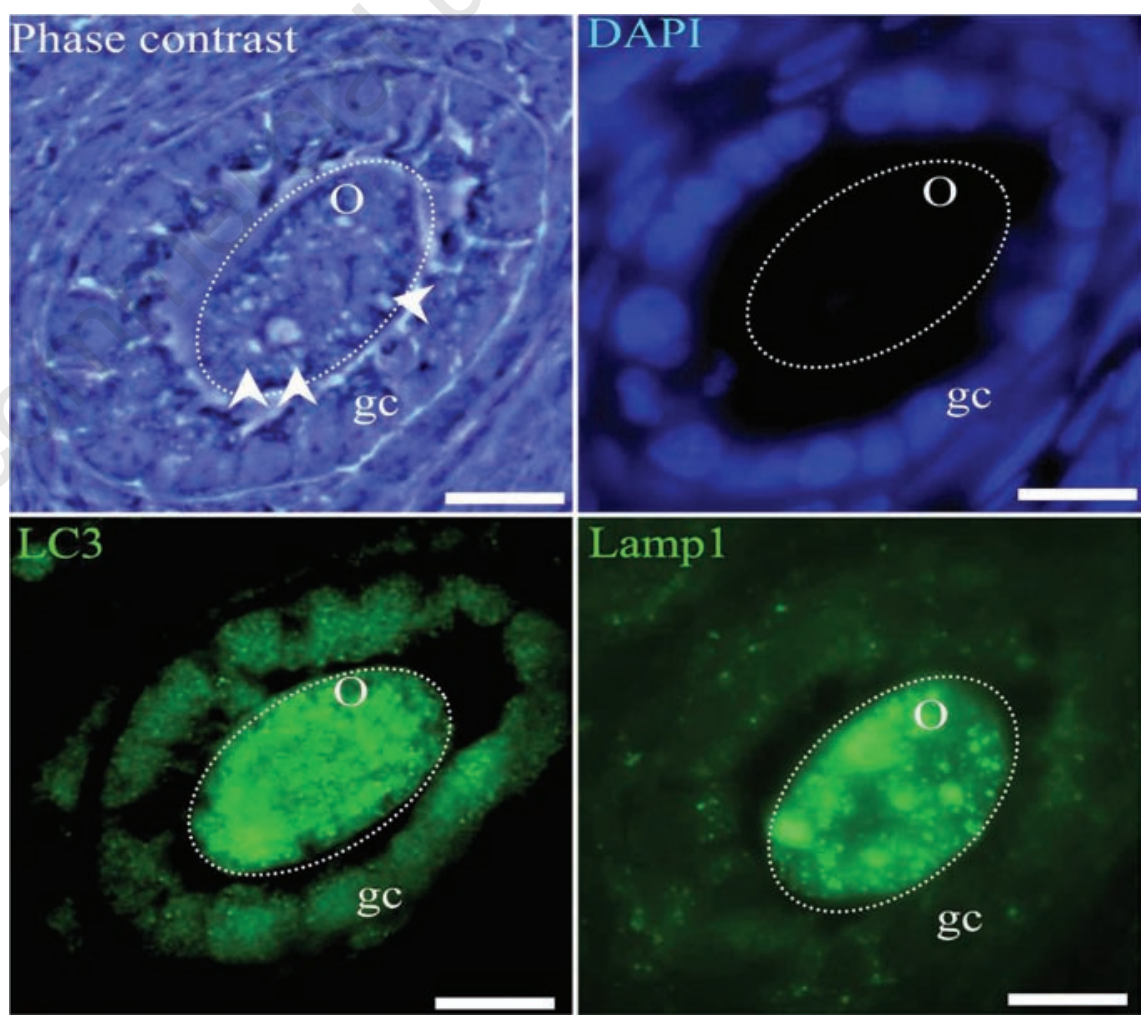

Figure 4. Atretic secondary follicles with an altered oocyte. The phase contrast image allows identification of regions with clear vesicles in the cytoplasm of the oocytes (arrow heads) and the altered shape of the follicle. The cytoplasm of the oocyte is intensively positive to Lamp1 and LC3 immunodetection (green fluorescence); indicating the autophagic process. The chromatin of the granulosa cells is labeled in blue using the DAPI staining method. Some of these cells are positively labeled by anti-Lamp1 or LC3 antibodies. The seriate sections, which do not pass through the nucleus, show the distribution of LC3 and Lamp1. The dotted circles indicate the approximate shape of the oocyte. o, oocyte; $g c$, granulosa cells. Scale bars: $20 \mu \mathrm{m}$. 

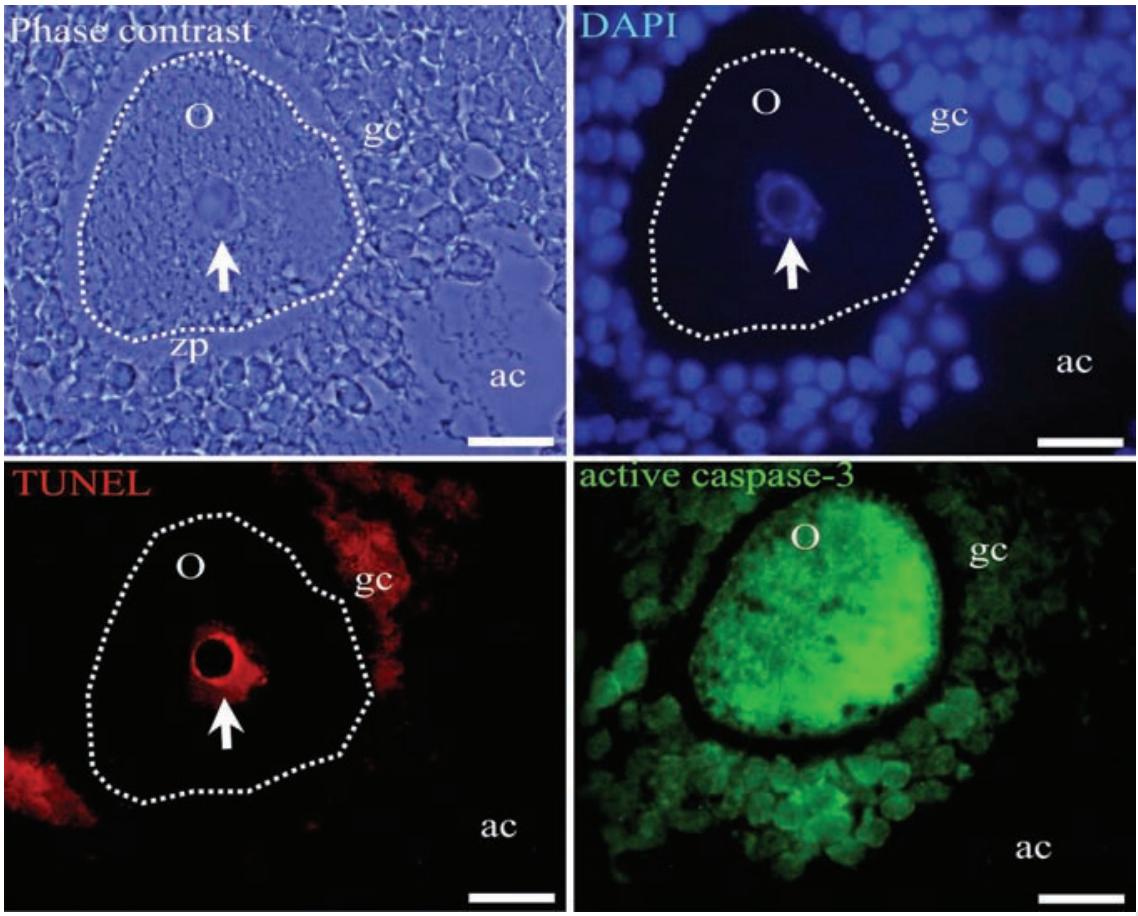

Figure 5. Antral atretic follicles with an altered oocyte. Phase contrast evidences several alterations, such as the irregular shape and condensed chromatin in the nucleus of the oocyte (arrow). The oocyte is simultaneously positive for active caspase- 3 immunodetection (green cytoplasm fluorescence) and the TUNEL technique (red nuclear fluorescence). The chromatin of the oocyte and the granulosa cells is shown by the blue fluorescence of the DAPI staining method. Some of granulosa cells are positive for active caspase-3 or TUNEL technique. Seriate section corresponding to active caspase-3 does not pass through the nucleus. $o$, oocyte; $z p$, zona pellucida; $g c$, granulosa cells; $a c$, antral cavity. The dotted line indicates the altered shape of the oocyte. Scale bars: $20 \mu \mathrm{m}$.

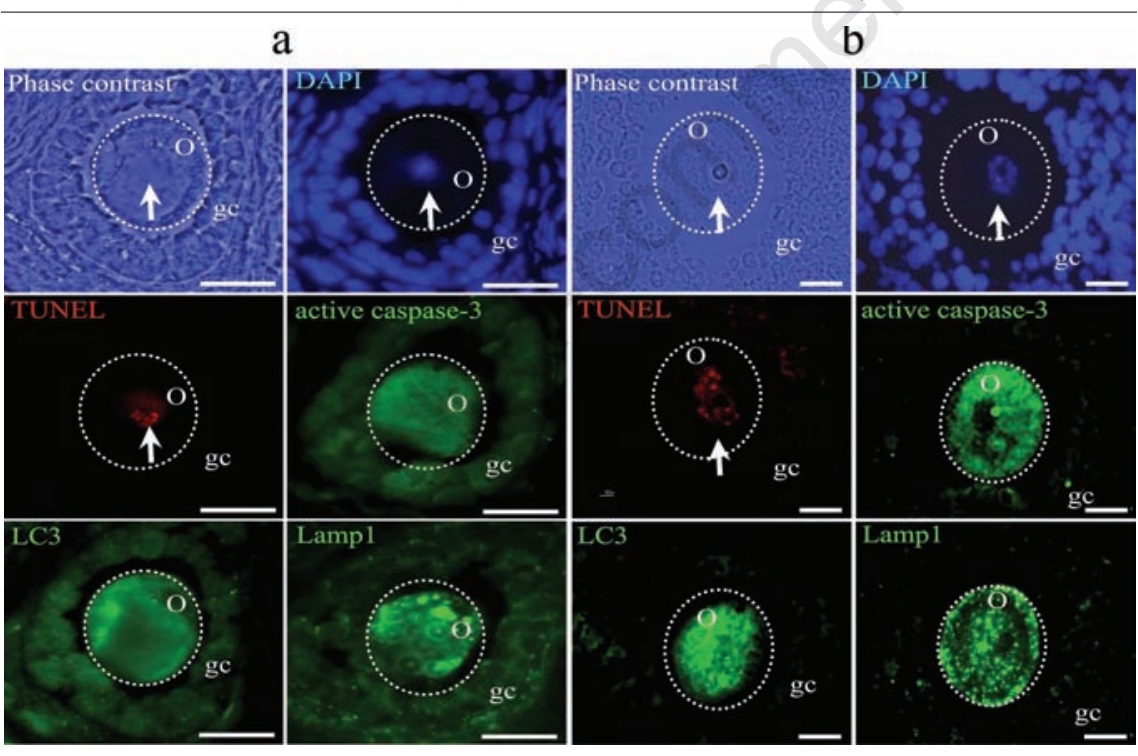

Figure 6. Atretic follicles with oocytes that are simultaneously positive for the pro-apoptotic and pro-autophagic markers. a) secondary follicle; b) antral follicle. In this oocyte, the four markers for the two different types of cell death are present: TUNEL (red fluorescence) and active caspase-3 (green fluorescence), which are markers of apoptosis; LC3 and Lamp1 (green fluorescence), which indicate autophagy. Phase contrast images show the morphology of the altered oocytes, and the presence of clear vesicles in different regions of the cytoplasm. DAPI (blue fluorescence) shows the distribution of the chromatin. The abundant labeling of the proteins identifying autophagic cell death is evident, as are the markers of apoptosis: active caspase- 3 and TUNEL. Arrows indicate the nuclei, which are positive to the DNA fragmentation identified by TUNEL positive. Some granulosa cells are positively labeled by active caspase-3, Lamp1, LC 3 antibodies or TUNEL technique. The dotted circle indicates the approximate shape of the oocyte. o, oocyte; $g c$, granulosa cells. Scale bars 20: $\mu \mathrm{m}$.

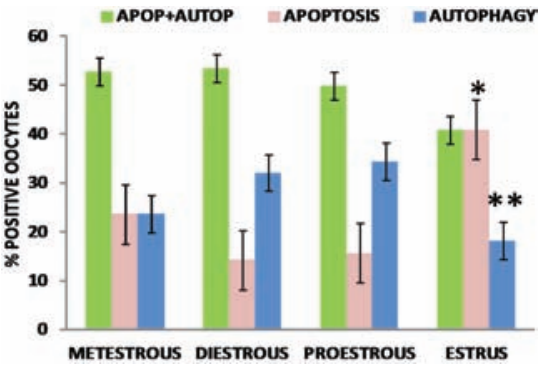

various procedures for evaluations at the molecular level. ${ }^{21-23}$ Those studies propose that apoptosis is an important component of the development and functioning of the ovary. In our study, we report some morphological characteristics of atretic follicles as detached granulosa cells, and alterations in the shape of oocytes. Moreover, the dying oocytes did not show the classical apoptotic features. Apoptosis is characterized by the activity of proteases called caspases. The major downstream effector of cell death is caspase-3, which in turn activates endogenous endonucleases responsible for DNA fragmentation. ${ }^{24}$ The biochemical features of apoptosis, including caspase activation, must be taken into account in any assessment of this process. The oocytes analyzed herein were positive for active caspase-3 and the TUNEL technique, two important markers for defining apoptosis. We found that the number of oocytes positive for apoptosis markers varied during the estrous cycle, with the highest number of dying oocytes that were positive for apoptosis markers being found during the estrus phase. The estrous cycle depends on the hormonal levels that exist in the ovary. In the estrus phase, FSH decreases, causing a reduction of the activity of FSH-dependent aromatase that limits the availability of estrogens and thus leads to a decrease in the proliferation of granulosa cells, and an increase in the amount of androgens, provoking irreversible follicular atresia. ${ }^{25}$ We found that in the proestrous stage, when estrogen levels are high ${ }^{26}$ the

METESTROUS DIESTROUS PROESTROUS ESTRUS

Figure 7. Percentage of oocytes positive for the different markers of apoptosis and autophagy. Combined apoptosis and autophagy is present simultaneously in more than $40 \%$ of the oocyte population in all phases. Oocytes undergoing only one process of cell death, apoptosis or autophagy, are present in all phases. Estrus is characterized by the lowest percentage of autophagy and the highest level of apoptosis. Error bars represent standard error. $(*)$ indicate the significant difference between the apoptosis level in the estrus phase and the others. $(* *)$ indicate the significant difference of autophagy level in the estrus phase and the others. The significant difference was obtained by a Student $t$-test $(P<0.05)$. 
number of oocytes in apoptosis is lower than in the metestrous and estrus phases. Recent evidences indicate an increased presence of the progesterone receptor membrane component1 (PGRMC1) protein during the proliferative phase of the estrous cycle. ${ }^{27}$ This receptor has been associated to proliferation events in normal $^{28}$ and cancer cells. ${ }^{29}$ Our results coincide with the anti- and pro-proliferative cellular activity promoted via the varying hormones levels through the estrous cycle. ${ }^{26}$

In addition to apoptosis, autophagy has also been described as a programmed type 2 cell death process; one in which caspases do not participate. The hallmark of autophagy is the high number of autophagic vesicles. Autophagy is a process through which cytoplasmic components, including macromolecules and organelles, are degraded by lysosomes..$^{30,31}$ As a physiological process, autophagy differs from autophagic cell death in the levels of intracellular degradation. Autophagic cell death involves higher lysosomal activity and an increased presence of proteins associated with autophagic vesicle formation. In mammals, lysosomes are the major digestive component and are responsible for breaking down foreign materials internalized by endocytosis and for destroying intracellular material delivered to lysosomes during autophagocytosis. $^{32}$

The microtubule-associated protein 1 light chain 3 (LC3), a homologue to yeast Atg8, is often used as an autophagosome marker. ${ }^{33}$ Considering that the intensity of autophagic activity levels differ in normal physiological processes and cell death processes, the quantification of the abundance of proteins that indicate the presence of lysosomes and autophagosomes, as Lamp1 and LC3, respectively, allowed us to characterize the events of cellular elimination. We found in normal oocytes low levels of these proteins evidencing basal autophagy. On the other hand, the atretic oocytes high levels of Lampl and LC3. Thus, we were able to identify the oocytes with high levels of LC3 and Lamp1 as cells in process of autophagic cell death.

We found that oocytes from adult ovaries present signs of both apoptotic and autophagic cell death, and that more than $40 \%$ of the oocytes that died in the estrous phase showed biochemical and morphological characteristics of both apoptosis and autophagy in the same cells. This means that these oocytes die through a process that involves a new form of cellular elimination.

We found that the markers of autophagic cell death, the LC3 and Lampl proteins, regulate germ line cell death during follicular atresia in adult rats; and that these proteins, together with the apoptotic activity of caspase3 , ultimately bring about DNA fragmentation.

a

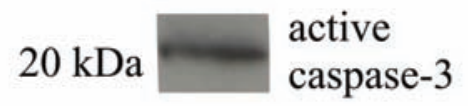

$$
\begin{array}{ll}
18 \mathrm{kDa} & \text { LC3-I } \\
16 \mathrm{kDa} & \text { LC3-II }
\end{array}
$$

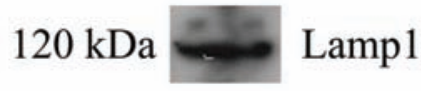

\section{b}
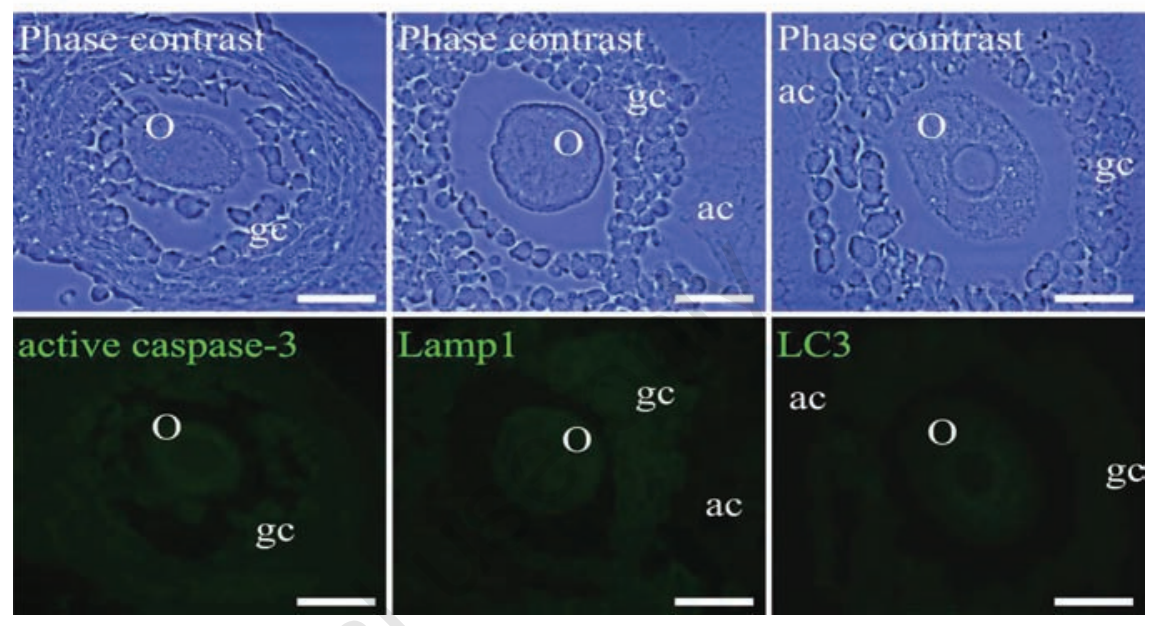

Figure 8. a) Western Blot analyses of the three different primary antibodies used in the present study. The image shows the different bands corresponding to: active caspase-3 ( $\approx 20 \mathrm{kDa})$, LC3-I and LC3-II (18 and $15 \mathrm{kDa}$ respectively), and Lamp1 ( $\approx 120 \mathrm{kDa}) ;$ b) atretic follicles of adult rat. The phase contrast shows the morphological characteristics of the follicle, the oocytes have an altered shape, the granulosa cells are detached of their neighbors and of the oocyte. The down panel is showing the absence of fluorescence in the tissue incubated without the primary antibody. o, oocyte; gc, granulosa cells; ac, antral cavity. Scale bars: $20 \mu \mathrm{m}$.
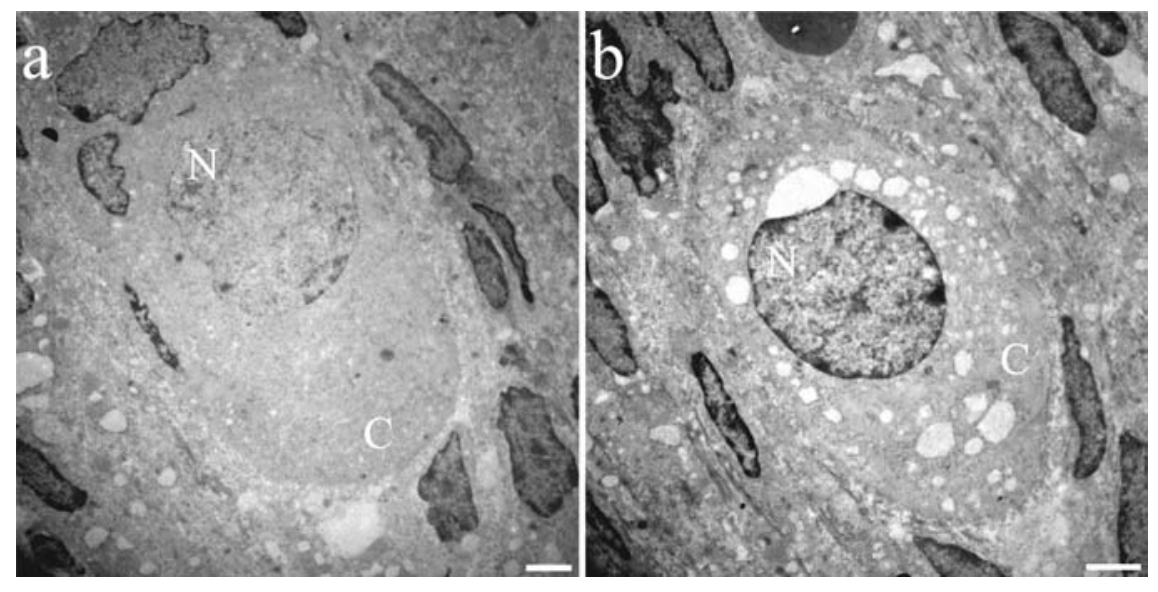

Figure 9. Electron microscopic images of primordial follicles of adult rats: a) normal oocyte, the cytoplasm is homogeneous, the shape is maintained, and the chromatin is distributed throughout the nucleus; b) oocyte in the process of dying with numerous autophagic vesicles in the cytoplasm and more compact chromatin than normal ones. The altered characteristics are observable in all the follicular development phases. $n$, nucleus; $c$, cytoplasm. Scale bars: $2 \mu \mathrm{m}$. 
The frequency of the simultaneous presence of the four markers in the same oocyte was higher than the frequency of the presence of only one marker of apoptosis or autophagy in an individual oocyte during the process of cell death. It is important to mention that the different patterns of cell death found in the oocytes were present in any stage of follicle development, because the evaluations of processes were carried out independently of the follicular phase.

The ultrastructural characterization of atresia in the adult rat allowed the identification of a correlation between morphological alterations and biochemical characteristics. The high frequency of the presence of LC3 and Lamp1 in the atretic oocytes coincides with an elevated number of small autophagic vesicles with cytoplasmic content and in diverse degrees of degradation. It is interesting to note that at low magnification the autophagosomes are not easily observed, but that at higher magnifications we were able to identify numerous autophagic vesicles distributed in the cytoplasm of the oocytes. The different morphological changes observed under light and electron microscopy in the atretic oocytes coincide with the presence of proteins that participate in apoptotic and autophagic cell death. These alterations are present in any phase of the follicular cycle since primordial, until pre-ovulatory follicles.

The levels of autophagy detected through the estrous cycle change according to the variations in estrogen levels, as shown by the finding that the proestrous is the phase with the highest estrogen level, ${ }^{26}$ and coincides with the highest number of oocytes that were positive for autophagic cell death. Lee et al. ${ }^{34}$ showed that estrogen supplementation in dormant blastocysts activates autophagy. In their model, autophagy is a mechanism used for the prolonged survival of dormant blastocysts, while in our study during follicular atresia in adult rats the increased estrogen allowed a greater autophagy which led oocytes into the autophagic cell death process. It is interesting to note the different roles of the autophagy under various stimuli, because it can function as a survival mechanism, ${ }^{34}$ but under a continuous estrogen stimulus the oocytes are led to the cell death via an autophagic process. In this work we found that numerous cells in autophagic cell death also show markers of apoptotic cell death.

In adult mammals, the development and survival of follicles relies on autocrine and paracrine signals involving growth factors of granulosa cells, theca cells, interstitial cells and oocytes. ${ }^{35-37}$ In this work, we show that elimination of the oocytes is caused not only by pro-apoptotic proteins but also by proautophagic ones, and that the features of the

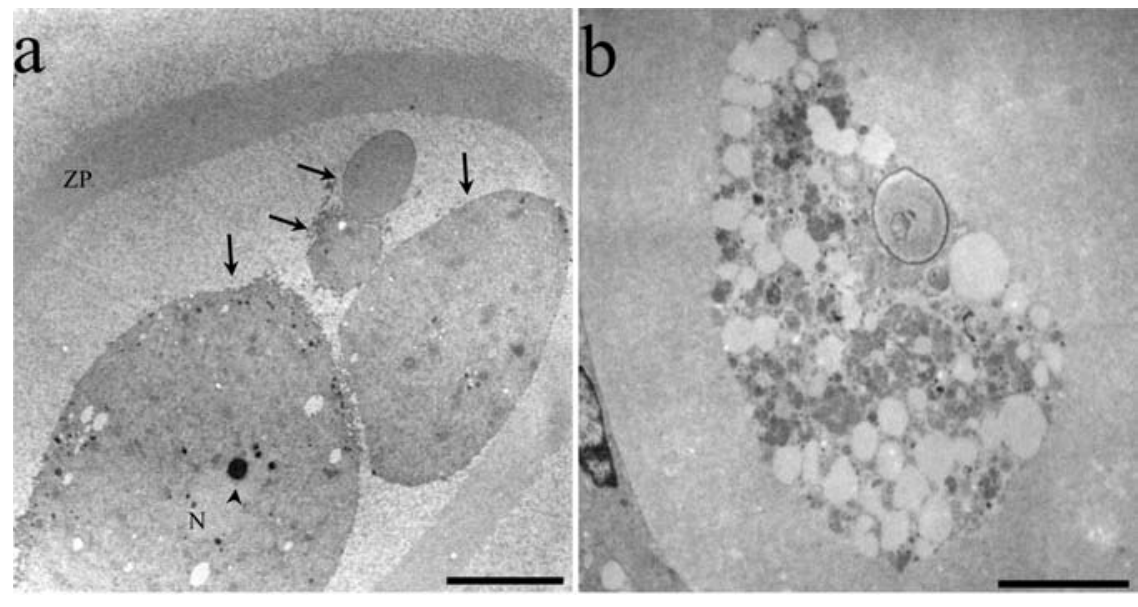

Figure 10. Ultrastructural features of atretic oocytes in antral follicles: a) a highly altered oocyte in the apoptotic process; the oocyte is fragmented (arrows) one of the cellular fragments has a substantially altered nucleus $(\mathrm{N})$ with a highly compacted nucleolus (arrowhead). The zona pellucida (ZP) is completely detached from the oocyte; b) oocyte in the autophagic process of cell death, evidenced by numerous autophagic vesicles in different degrees of degradation. Uranyl acetate and lead citrate. Scale bars: a, 10 microns; b, $5 \mu \mathrm{m}$.
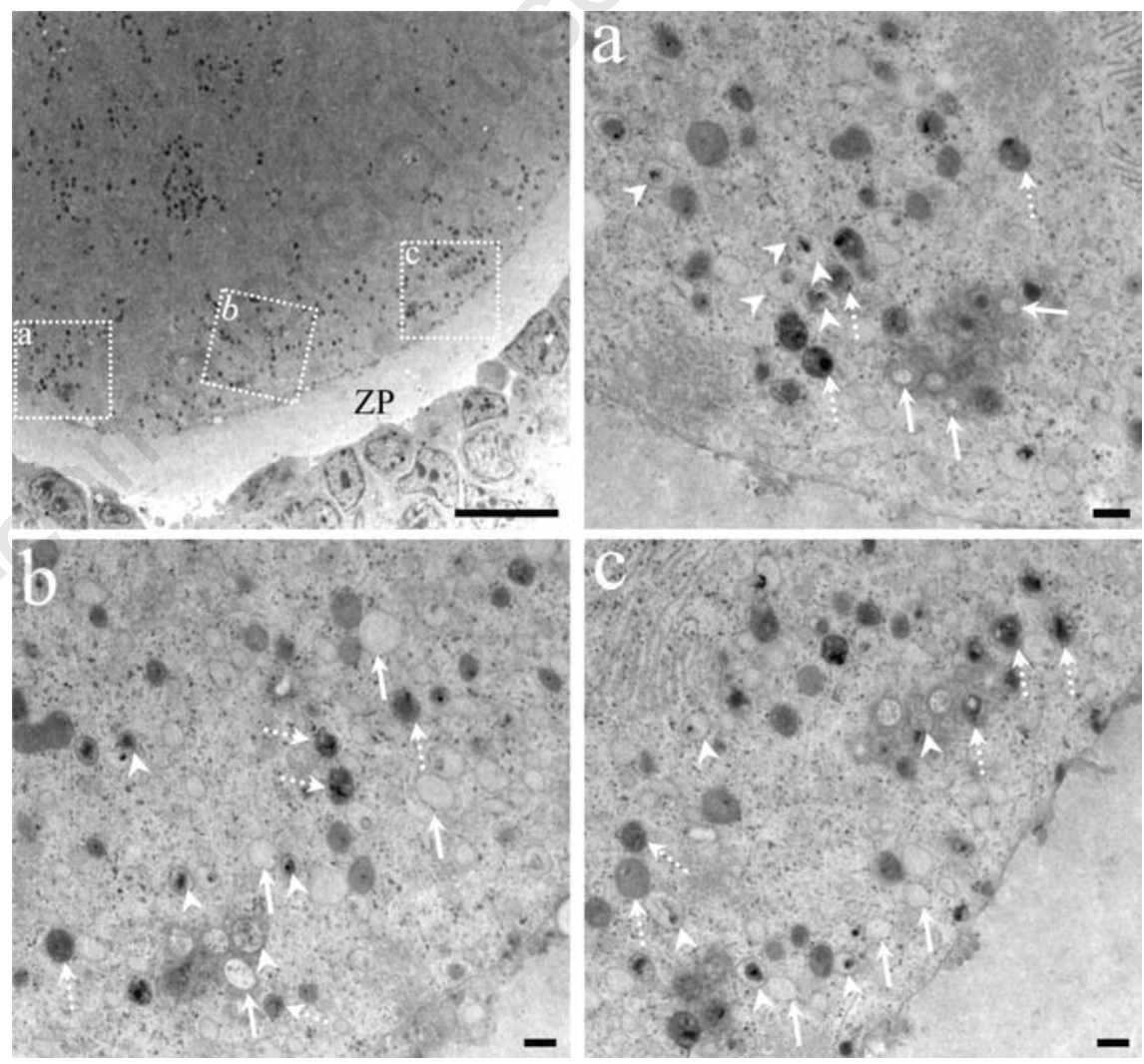

Figure 11. Ultrastructural features of an oocyte in the autophagic cell death process. The relationship between the granulosa cells and the oocyte are highly altered; there are no cytoplasmic prolongations of the granulosa or microvillus of the oocyte. The zona pellucida (ZP) surrounds a highly altered oocyte with numerous dense structures. The dotted squares have been enlarged in micrographs $\mathrm{a}, \mathrm{b}$ and $\mathrm{c}$. High magnification pictures of autophagic vesicles in different degrees of degradation of their content. Arrows point to clear autophagic vesicles containing highly degraded material; arrowheads point to autophagic vesicles containing debris in an intermediate degree of degradation. The autophagic vesicles with recently sequestered material are indicated by dotted arrows. Scale bars: low magnification 10 microns; a, b, and c, $500 \mathrm{~nm}$. 


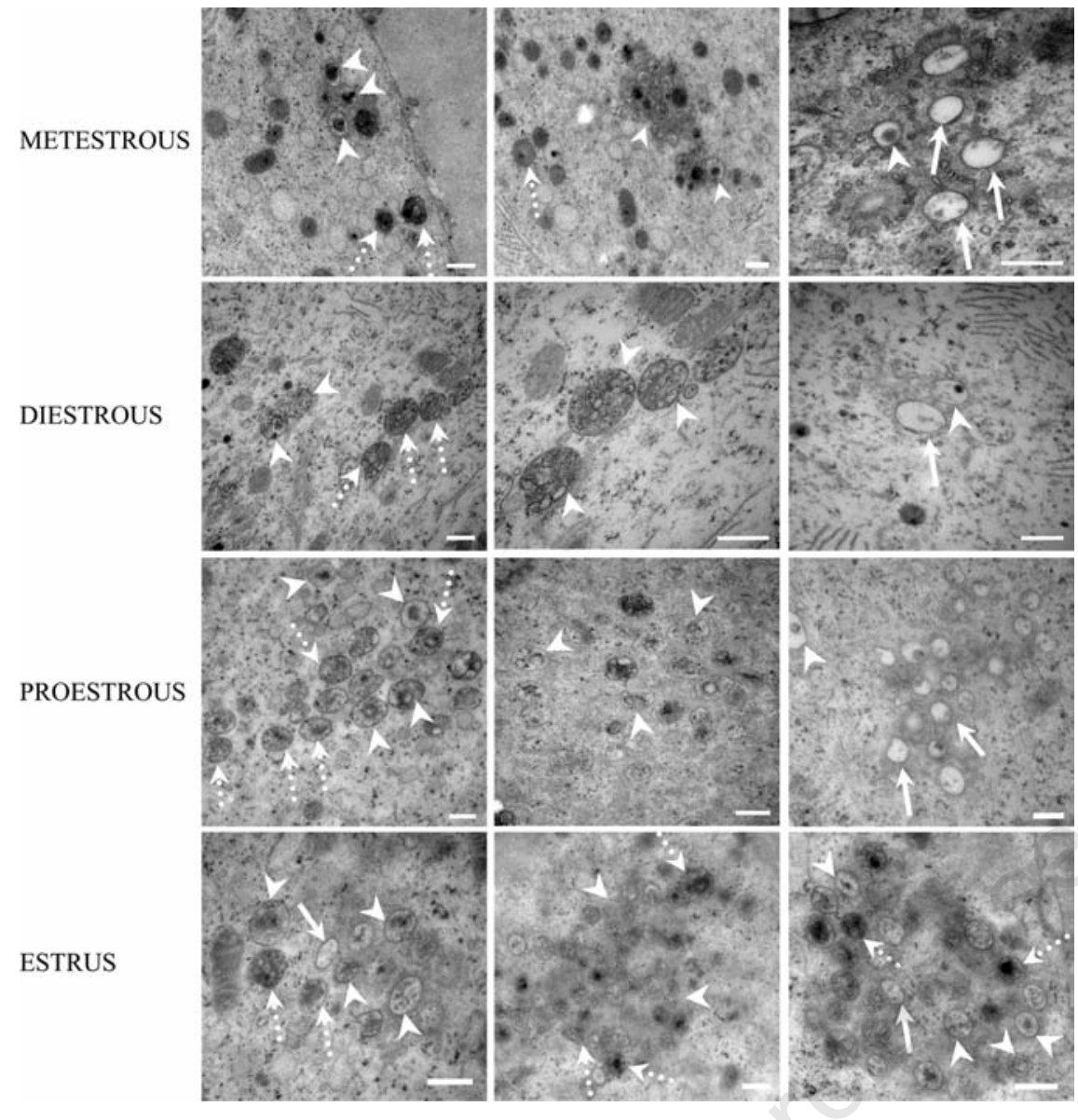

Figure 12. Electron micrographs of the cytoplasm of atretic oocytes of adult rats showing autophagic vesicles in different degrees of degradation in all phases of the estrous cycle. Dotted arrows indicate autophagic vesicles in the initial phase of degradation; arrowheads show autophagosomes with an intermediate stage of degradation of their content. Arrows point to clear vesicles containing material in the last phase of autophagic degradation. Scale bars: $500 \mathrm{~nm}$.

process of oocyte death change in different stages of the estrous cycle. The high levels of estrogens that stimulate cell proliferation coincide with low levels of cell death detected in the proestrous phase. Cells under the influence of cell-proliferation-inhibiting hormones are eliminated massively through the participation of the proteins involved in apoptosis and autophagy. In vitro studies have demonstrated that LC3 and Atg7 associated with autophagy, are activated by hormonal changes. $^{34}$

The development of the oocyte is a critical aspect of follicular development because in normal follicles there is a bidirectional communication of cytoplasmic signals between the oocyte and the granulosa cells that involves an interchange of nutrients, second messengers and other signals that coordinate the metabolisms of both cell types. ${ }^{38}$ These findings evidence the importance of studying the events leading to oocyte death. Various studies on cell death indicate that features of apoptosis and autophagy can be found simultaneously in the same cells stimulated in vitro to express the proteins of only one of these processes of cell death. ${ }^{39}$ In the present work, we studied ovaries of normal rats in which cell death is a normal event during follicular atresia, and showed that different mechanisms of cell death may be present simultaneously in the same oocyte and together could make up a more efficient system of cell elimination. These results indicate that the pro-autophagic and pro-apoptotic proteins control the elimination of altered oocytes, and that the linking of these machineries to pathways of cell death directs the spatio-temporal activation of oocyte cell death in adulthood.

Our observations contribute to a better understanding of the massive elimination of cells during follicular atresia and may open new lines of research leading to the development of new procedures to attack malignant cells that do not respond to pro-apoptotic stimuli by stimulating autophagic cell death.
Understanding of the routes of cell death and, moreover, knowledge of the processes of oocyte death, are important for treating certain pathologies, since in many cases diseased cells are not responsive to the induction of apoptosis, making it necessary to induce alternative signaling pathways, or to employ combined strategies. The knowledge that the cells have the machinery necessary to undergo both of cell death routes opens the possibility to stimulate cellular elimination by activating several metabolic pathways.

\section{References}

1. Baker TG. A quantitative and cytological study of germ cells in the human ovaries. Proc Roy Soc Lond 1963;158:417-33.

2. Braw RH, Byskov AG, Peters H, Faber M. Follicular atresia in the human infant ovary. J Reprod Fertil 1976;46:55-9.

3. Morita Y, Tilly JL. Oocyte apoptosis: like sand through an hourglass Dev Biol 1999;213:1-17.

4. Richards JS. Maturation of ovarian follicles: action and interactions of pituitary and ovarian hormones on follicular cell differentiation. Physiol Rev 1980;60:51-89.

5. Byskov AG. Cell kinetic studies of follicular atresia in the mouse ovary. J Reprod Fertil 1974; 37:277-85.

6. Kerr JFR, Wyllie AH, Currie AR. Apoptosis: a basic biological phenomenon with wide ranging implications in tissue kinetics. $\mathrm{Br}$ J Cancer 1972;26:239-57.

7. Thornberry NA, Lazebnik Y. Caspases: enemies within. Science 1998;281:1312-6.

8. Mizushima N, Ohsumi Y, Yoshimori T. Autophagosome formation in mammalian cells. Cell Struct Funct 2002;27:421-9.

9. Klionsky DJ. The molecular machinery of autophagy: unanswered questions. J Cell Sci 2005;118:7-18.

10. Kabeya Y, Mizushima N, Ueno T, Yamamoto A, Kirisako T, Noda T, et al. LC3, a mammalian homologue of yeast Apg8p, is localized in autophagosome membranes after processing. EMBO J 2000;19:5720-8.

11. Kroemer G, Jaattela M. Lysosomes and autophagy in cell death control. Nat Rev Cancer 2005;5:886-97.

12. Tilly JL, Tilly KI, Perez GI. The genes of cell death and cellular susceptibility to apoptosis in the ovary: a hypothesis. Cell Death Differ 1997;4:180-7.

13. Maravei DV, Morita Y, Kuida K, Tilly JL. Pre- and postnatal ovarian apoptosis defects in caspase-9-deficient mice. Mol Biol Cell (Suppl) 1999; 10:352a

14. Bergeron L, Perez GI, Mcdonald G, Shi L, Sun Y, Jurisicova A, et al. Defects in regu- 
lation of apoptosis in caspase-2-deficient mice. Genes Dev 1998; 12:1304-14.

15. Exley GE, Tang C, McElhinny AS, Warner CM. Expression of caspase and BCL-2 apoptotic family members in mouse preimplantation embryos. Biol Reprod 1999;61:231-9.

16. Perez GI, Tao XJ, Tilly JL. Fragmentation and death (a.k.a. apoptosis) of ovulated oocytes. Mol Hum Reprod 1999;5:414-20.

17. Ortiz R, Echeverría OM, Salgado R, Escobar ML, Vázquez-Nin GH. Apoptosis Fine structural and cytochemical analysis of the processes of cell death of oocytes in atretic follicles in new born and prepubertal rats. Apoptosis 2006;11:25-37.

18. Escobar ML, Echeverría OM, Ortíz R, Vázquez-Nin GH. Combined apoptosis and autophagy, the process that eliminates the oocytes of atretic follicles in immature rats. Apoptosis 2008;13:1253-66.

19. Escobar ML, Echeverría OM, SánchezSánchez L, Méndez C, Pedernera E, Vázquez-Nin GH. Analysis of different cell death processes of prepubertal rat oocytes in vitro. Apoptosis 2010;15:511-26.

20. Abramoff MD, Magalhaes PJ, Ram SJ. Image processing with ImageJ. Biophotonics International 2004;11:36-42.

21. Hughes FM, Gorospe WC. Biochemical identification of apoptosis (programmed cell death) in granulosa cells evidence for a potential mechanism underlying follicular atresia. Endocrinology 1991;129:2415 22.

22. Hsueh AJW, Billig H, Tsafriri A. Ovarian follicle atresia: a hormonally controlled apoptotic process. Endocr Rev 1994;15:
707-24.

23. Hussein MR. Apoptosis in the ovary: molecular mechanisms. Hum Reprod Update 2005;11:162-78.

24. Wyllie AH, Arends MJ, Morris RG, Walker SW, Evan G. The apoptosis endonuclease and its regulation. Semin Immunol 1992;4:389-97.

25. Tesone M, Abramovich D, Irusta G, Parborell F. Autocrine and paracrine regulation of the ovary. In: PJ Chedrese (ed.) Reproductive endrocrinology. A molecular approach. Springer, New York, USA, 2009, pp. 241-8.

26. Haruyama N, Igarashi K, Saeki S, OtsukaIsoya M, Shinoda $\mathrm{H}$, Mitani $\mathrm{H}$. Estrouscycle-dependent variation in orthodontic tooth movement. J Dent Res 2002;81:40610.

27. Luciano AM, Corbani D, Lodde V, Tessano I, Francios F, Peluso JJ, et al. Expression of progesterone receptor membrane component-1 in bovine reproductive system during estrous cycle. Eur J Histochem 2011;55:e27.

28. Lodde V, Peluso JJ. A novel role for progesterone and progesterone receptor membrane component 1 in regulating spindle microtubule stability during rat and human ovarian cell mitosis. Biol Reprod 2011;84:715-22.

29. Peluso JJ. Progesterone signaling mediated through progesterone receptor membrane component-1 in ovarian cells with special emphasis on ovarian cancer. Steroids 2011;76:903-9.

30. Mizushima N. Autophagy: process and function. Genes Dev 2007;21:2861-73.
31. Levine B, Kroemer G. Autophagy in the pathogenesis of disease. Cell 2008;132:2742.

32. de Duve C. Lysosomes revisited. Eur J Biochem 1983; 37:391-7.

33. Klionsky DJ, Baehrecke EH, Brumell JH, Chu CT, Codogno P, Cuervo AM, et al. A comprehensive glossary of autophagyrelated molecules and processes (2nd edition). Autophagy 2011;7:1273-94.

34. Lee JE, Oh HA, Song H, Jun JH, Roh CR, $\mathrm{Xie} \mathrm{H}$, et al. Autophagy regulates embryonic survival during delayed implantation. Endocrinology 2011;152:2067-75.

35. Drouilhet L, Taragnat C, Fontaine J, Duittoz A, Mulsan P, Bodin L, et al. Endocrine Characterization of the Reproductive Axis in Highly Prolific Lacaune Sheep Homozygous for the FecLL Mutation. Biol Reprod 2010;82:815-24.

36. Chun SY, Eisenhauer KM, Minami S, Billig H, Perlas E, Hsueh AJ. Hormonal regulation of apoptosis in early antral follicles: follicle-stimulating hormone as a major survival factor. Endocrinology 1996;137: 1447-56.

37. Hsueh AJ, Billig H, Tsafriri A. Ovarian follicle atresia: a hormonally controlled apoptotic process. Endocr Rev 1994;15:707-24.

38. Buccione R, Schroeder AC, Eppig JJ. Interactions between somatic cells and germ cells throughout mammalian oogenesis Biol Reprod 1990;43:543-7.

39. Fimia GM, Piacentini M. Regulation of autophagy in mammals and its interplay with apoptosis. Cell Mol Life Sci 2010;67: 1581-8. 